\title{
Numerical modelling of the flow in the resin infusion process on the REV scale: A feasibility study
}

Jabbaribehnam, Mirmasoud; Jambhekar, V. A.; Gersborg, A. R.; Spangenberg, Jon; Hattel, Jesper Henri; Helmig, R.

\section{Published in:}

A I P Conference Proceedings Series

Link to article, DOI:

$10.1063 / 1.4951807$

Publication date:

2016

Document Version

Publisher's PDF, also known as Version of record

Link back to DTU Orbit

Citation (APA):

Jabbaribehnam, M., Jambhekar, V. A., Gersborg, A. R., Spangenberg, J., Hattel, J. H., \& Helmig, R. (2016). Numerical modelling of the flow in the resin infusion process on the REV scale: A feasibility study. $A$ I $P$ Conference Proceedings Series, 1738, [030051]. https://doi.org/10.1063/1.4951807

\section{General rights}

Copyright and moral rights for the publications made accessible in the public portal are retained by the authors and/or other copyright owners and it is a condition of accessing publications that users recognise and abide by the legal requirements associated with these rights.

- Users may download and print one copy of any publication from the public portal for the purpose of private study or research.

- You may not further distribute the material or use it for any profit-making activity or commercial gain

- You may freely distribute the URL identifying the publication in the public portal 


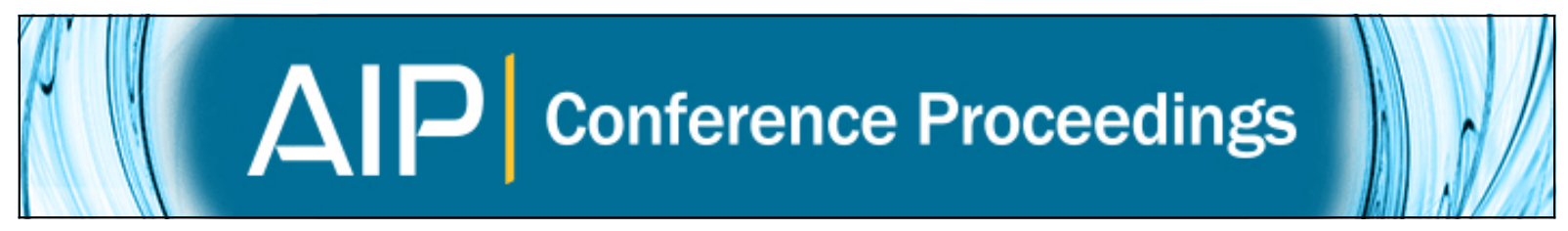

\section{Numerical modelling of the flow in the resin infusion process on the REV scale: A} feasibility study

M. Jabbari, V. A. Jambhekar, A. R. Gersborg, J. Spangenberg, J. H. Hattel, and R. Helmig

Citation: AIP Conference Proceedings 1738, 030051 (2016); doi: 10.1063/1.4951807

View online: http://dx.doi.org/10.1063/1.4951807

View Table of Contents: http://scitation.aip.org/content/aip/proceeding/aipcp/1738?ver=pdfcov

Published by the AIP Publishing

\section{Articles you may be interested in}

Comparative study of numerical models of the laser forming process

J. Laser Appl. 27, S29105 (2015); 10.2351/1.4907397

Numerical Modeling of Fluid Flow in the Tape Casting Process

AIP Conf. Proc. 1389, 143 (2011); 10.1063/1.3636690

Stochastic Flow Modeling for Resin Transfer Moulding

AIP Conf. Proc. 1152, 284 (2009); 10.1063/1.3203279

A Parallel Computational Environment for Modeling the Resin Transfer Molding Process AIP Conf. Proc. 712, 305 (2004); 10.1063/1.1766541

An Overview of Multi-Scale Flow/Thermal/Cure Modeling for Resin Transfer Molding Processes of Complex Structural Composites

AIP Conf. Proc. 712, 135 (2004); 10.1063/1.1766513 


\title{
Numerical Modelling of the Flow in the Resin Infusion Process on the REV Scale: A Feasibility Study
}

\author{
M. Jabbari*, V.A. Jambhekar ${ }^{\dagger}$, A.R. Gersborg**, J. Spangenberg*, J.H. Hattel ${ }^{*}$ and \\ R. Helmig ${ }^{\dagger}$ \\ *Process Modelling Group, Department of Mechanical Engineering, Technical University of Denmark, Nils \\ Koppels Allé, 2800 Kgs. Lyngby, Denmark. \\ ${ }^{\dagger}$ Department of Hydromechanics and Modelling of Hydrosystems, Institute for Modelling Hydraulic and \\ Environmental Systems, Universität Stuttgart, Stuttgart, Germany. \\ ${ }^{* *}$ SCION DTU, Diplomvej 373N, DK-2800 Lyngby, Denmark
}

\begin{abstract}
The resin infusion process (RIP) has developed as a low cost method for manufacturing large fibre reinforced plastic parts. However, the process still presents some challenges to industry with regards to reliability and repeatability, resulting in expensive and inefficient trial and error development. In this paper, we show the implementation of 2D numerical models for the RIP using the open source simulator $\mathrm{DuMu}^{\mathrm{X}}$. The idea of this study is to present a model which accounts for the interfacial forces coming from the capillary pressure on the so-called representative elementary volume (REV) scale. The model is described in detail and three different test cases — a constant and a tensorial permeability as well as a preform/Balsa domain - are investigated. The results show that the developed model is very applicable for the RIP for manufacturing of composite parts. The idea behind this study is to test the developed model for later use in a real application, in which the preform medium has numerous layers with different material properties.
\end{abstract}

Keywords: Resin infusion, fluid flow, porous media, REV scale PACS: 81.05.Je, 02.60.Lj, 47.56.+r, 83.10.-y

\section{INTRODUCTION}

The resin infusion process (RIP) or vacuum assisted resin transfer moulding (VARTM), a.k.a VI, VM, RIFT, is widely used as an alternative to open mould techniques to produce large components [1]. Resin infusion (RI) is part of the liquid composite moulding (LCM) process family. The term LCM describes the closed mould processes in which a liquid polymeric resin is impregnated through a fibrous reinforcement (e.g. see Figure 1(a)). This process offers numerous cost advantages over traditional resin transfer molding via lower tooling costs, room temperature processing, and scalability to large structures [2].

As shown in Figure 1(a), layers of fibrous reinforcement are laid on the mould to form the preform. A peel ply is then laid over the preform, in order to ease separation of the consumables from the part, and to obtain a reasonable surface on the side of the part that is not in contact with the mould. A distribution medium (resin feed line) is positioned over the peel ply to enhance the resin flow if the preform has low in-plane permeability. Once inlet and vent tubes are placed, the mould is covered with a vacuum bag sealed with sealant tape [3].

The aim of this study is to develop a global solver to simulate the resin flow in a porous body which accounts for the interfacial forces coming from the capillary pressure. The new model concept can be used to describe isothermal twophase flow in a porous medium, and it is based on existing approaches and valid on the representative elementary volume (REV) scale [4]. In the following, the developed model concept will be briefly explained including its implementation into the numerical simulator $\mathrm{DuMu}^{\mathrm{X}}[5]$.

\section{NUMERICAL MODEL}

\section{Model Concept}

Flow and transport in porous media are relevant to many areas of engineering and science including composite manufacturing, groundwater hydrology, oil and gas recovery, membrane separations, catalyst supports, and biological 
scaffolds [4]. These applications cover a vast range of length scales; from the kilometer scale in oil and gas recovery to the micron scale for micro-fluidic devices. In all of these examples, a quantitative prediction of flow and transport on strongly varying spatial and temporal scales is required and typically achieved with mathematical and numerical models [6].

The model concept for the RIP is shown in Figure 1(b), where a free flow region with high porosity and high permeability value, $\left\{\mathbf{K}_{1}, \phi_{1}\right\}^{h_{1}}$, stands on top of the porous medium, $\left\{\mathbf{K}_{2}, \phi_{2}\right\}^{h_{2}}$, in which $h_{1} \ll h_{2}$. It should be noted that in the case where different preform layers exist in the porous medium, each layer will be characterized by its properties as $\left\{\mathbf{K}_{i}, \phi_{i}\right\}^{h_{i}}$. The flow in the distribution medium is assumed to be laminar $(R e \ll 1)[1,2]$.

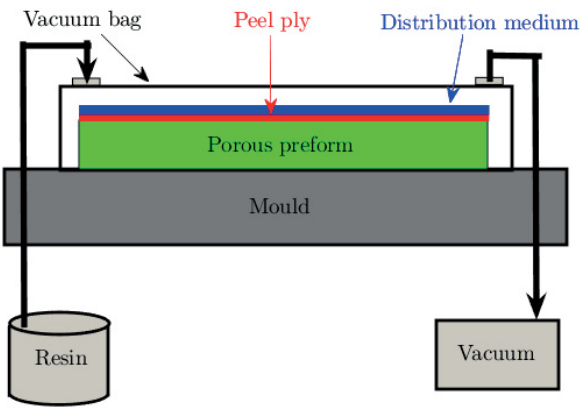

(a) (b)

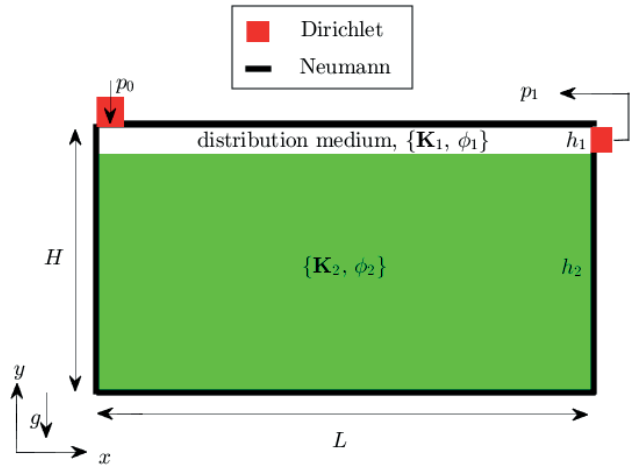

FIGURE 1. (a) General schematic illustration of RIP, and (b) model concept with a two-phase flow in the porous medium.

\section{Mathematical Model}

In case of a two-phase model ( $\left.\operatorname{DuMu}^{\mathrm{X}}: 2 \mathrm{p}\right)$, the following mass balance equation is solved for both the wetting resin phase $r$ and the non-wetting air phase $a$

$$
\frac{\partial\left(\rho_{\alpha} \phi S_{\alpha}\right)}{\partial t}+\nabla \cdot\left(\rho_{\alpha} \mathbf{q}_{\alpha}\right)=0, \quad \alpha \in\{r, a\}
$$

where $\rho_{\alpha}$ is the density of the fluid, $\phi$ is porosity, $S_{\alpha}$ is fluid saturation, and $\mathbf{q}_{\alpha}$ is the volumetric flux vector for phase $\alpha$. The propagation of the two phases is obtained by coupling equation (1) with a relationship that relates the saturation to the capillary pressure, equation (2), a closure condition for the saturation, equation (3), a relationship that relates volumetric fluxes to pressure gradients via a multi-phase version of Darcy's law, equation (4), and an expression for the relative permeabilities, equation (5),

$$
\begin{gathered}
S_{\alpha}=S_{\alpha}\left(p_{c}\right)=S_{\alpha}\left(p_{a}-p_{r}\right) \\
S_{a}+S_{r}=1 \\
\mathbf{q}_{\alpha}=-\frac{k_{r \alpha}}{\mu_{\alpha}} \cdot \mathbf{K}\left(\nabla p_{\alpha}-\rho_{\alpha} \mathbf{g}\right) \\
k_{r \alpha}=k_{r \alpha}\left(S_{\alpha}\right)
\end{gathered}
$$

where $p_{c}$ is the capillary pressure, $p_{\alpha}$ is the individual phase pressures, $\mathbf{K}$ is the intrinsic permeability tensor, $\mu_{\alpha}$ is the viscosity of phase $\alpha, \mathbf{g}$ is the gravitational vector, and $k_{r \alpha}$ is the relative permeability to phase $\alpha$ which is a nonlinear function of the saturation. Given this set of equations, boundary and initial conditions must be supplied to close the system of equations and get a unique solution. These are usually given as known pressures, saturations or fluxes in each of the fluid phases [7].

Choosing the proper constitutive laws is one of the key issues when it comes to solve the fluid flow of a material. In our case, the constitutive laws are given by equations (2) and (5). For the relative permeability $k_{r \alpha}$ of a phase $\alpha$ which is a function of the phase saturation $S_{\alpha}$, the well-known Brooks-Corey [8] model is used: 


$$
k_{r w}=S_{e}^{\left(\frac{2+3 \lambda}{\lambda}\right)}, \quad k_{r n}=\left(1-S_{e}\right)^{2}+1-S_{e}^{\left(\frac{2+\lambda}{\lambda}\right)}
$$

where $S_{e}=\frac{S_{r}-S_{r r}}{1-S_{r r}}$ is the effective saturation with the residual saturation $S_{r r}$, and $\lambda$ is the Brooks-Corey parameter that has to be determined experimentally. The capillary pressure for the resin is $p_{c}\left(S_{r}\right)=p_{a}-p_{r}$, and the pressuresaturation relationship is again given by Brooks-Corey [8] model

$$
S_{e}\left(p_{c}\right)=S_{e}=\frac{S_{r}-S_{r r}}{1-S_{r r}}=\left(\frac{p_{d}}{p_{c}}\right)^{\lambda}
$$

where $p_{d}$ is the entry pressure, $S_{e}$ is the effective saturation, $S_{r r}$ is the residual resin saturation, and $\lambda$ is the BrooksCorey parameter. All the parameters mentioned for the Brooks-Corey model are dependent on the porous medium.

\section{Numerical Implementation}

The implementation of the numerical scheme has been conducted in the modelling toolbox $\mathrm{DuMu}^{\mathrm{X}}$, which is a free and open-source simulator for flow and mass transport in porous media, based on the Distributed and Unified Numerics Environment (DUNE) [9]. Its main intention is to provide a sustainable and consistent framework for the implementation and application of model concepts, constitutive relations, discretizations and solvers [5].

\section{RESULTS AND DISCUSSION}

Initially, the resin fluid has been added to the material database with the density and viscosity values of $\rho_{r}=1090$ $\left[\mathrm{kg} / \mathrm{m}^{3}\right]$ and $\mu_{r}=0.14[\mathrm{~Pa} \cdot \mathrm{s}]$, receptively. The constant inlet injection pressure is assumed to $p_{0}=85000[\mathrm{~Pa}]$, while the vacuum pressure is set be zero $\left(p_{1}=0[\mathrm{~Pa}]\right)$. The porosity values are assumed to be $\phi_{1}=0.9$ and $\phi_{2}=0.4$. In the simulation domain we use $L=0.2[\mathrm{~m}], H=0.102[\mathrm{~m}], h_{1}=0.002[\mathrm{~m}]$, and $h_{2}=0.1[\mathrm{~m}]$, where $h_{2} / h_{1}=50$.

In the first case study, a constant permeability of $\mathbf{K}_{1}=8.8 \times 10^{-8}\left[\mathrm{~m}^{2}\right]$ and $\mathbf{K}_{2}=2 \times 10^{-12}\left[\mathrm{~m}^{2}\right]$ are chosen for the distribution medium and the preform region, respectively. In addition, the Brooks-Corey model with an entry pressure of $p_{d}=100[\mathrm{~Pa}], S_{r r}=0$, and $\lambda=2$ is used. The results of simulation are shown in Figure 2(a), and they are similar to the results presented in the studies $[1,2,3]$. As seen in the figure, the resin fast fills up the distribution medium region, and then starts to impregnate the preform region. This effect is due to the relative low resistance (high permeability) in the distribution medium as compared to the preform region. The pressure boundary condition imposes a pressure gradient in the distribution medium which explain the difference in propagation speed of the flow front at the left and right boundary of the domain.

(a)
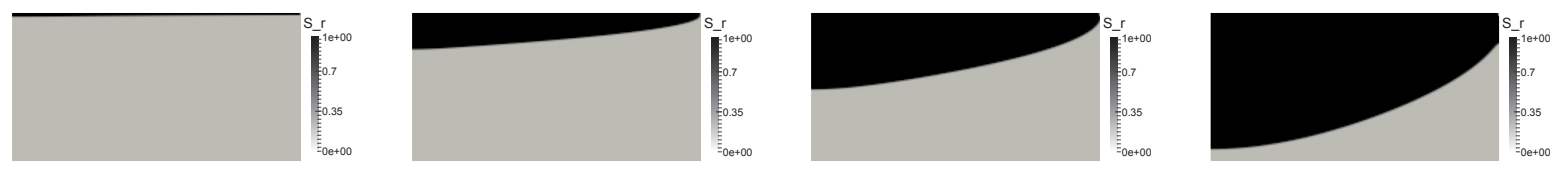

(b)
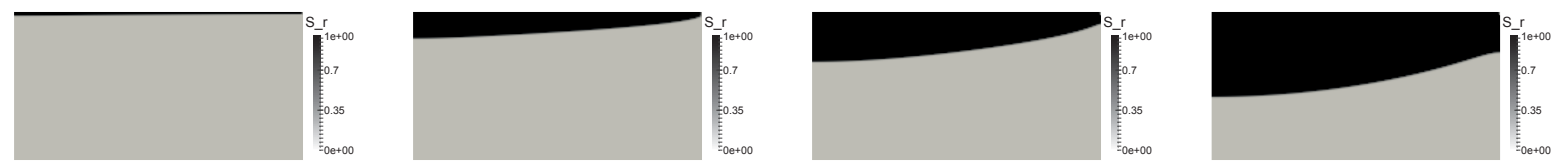

FIGURE 2. Contour of resin saturation (S_r) for (a) constant, and (b) tensorial, permeability in the preform region. The results are shown in the same time instinct.

In the other study, we have implemented a tensorial permeability for the preform medium

$$
\mathbf{K}_{2}=\left[\begin{array}{ll}
K_{2 x x} & K_{2 x y} \\
K_{2 y x} & K_{2 y y}
\end{array}\right]=\left[\begin{array}{cc}
6 \times 10^{-12} & 1.0 \times 10^{-18} \\
1.0 \times 10^{-18} & 1 \times 10^{-12}
\end{array}\right]\left[\mathrm{m}^{2}\right]
$$

where $K_{2 x x}$ is the permeability of the preform layer in the longitudinal $(x)$ direction, and $K_{2 y y}$ is the permeability of the preform layer in the thickness $(y)$ direction. For the sake of numerical simplicity we avoid putting zero values for $K_{2 x y}=K_{2 y x}$, and set them to a low number. 
The results of this simulation are illustrated in Figure 2(b). Here, it is seen that due to the higher flow resistance (lower permeability) in the $y$ direction, the resin flows less in the thickness direction of the preform. This fact becomes evident when comparing the results with the ones for a constant permeability (comparing Figure 2(a) and (b)). Based on this comparison, it is also concluded that the filling time for the tensorial case will be higher than when using the constant permeability (comparing the volume filled by resin).

In the last case study, we have included a region inside the preform domain that has a different permeability and porosity value, e.g. see Figure 3. The idea behind this study is to test the developed model for later use for the real application, in which the preform medium has different layers with different material properties. For this particular study, we assume that the third region has the characteristic properties of a Balsa wood (see Figure 3(a)) which is normally used as a core material in the manufacturing of wind turbine blades. The properties of the Balsa wood is chosen to be $\phi_{3}=0.13$ and $\mathbf{K}_{3}=4.42 \times 10^{-11}\left[\mathrm{~m}^{2}\right]$ [10], and the dimensions are $h_{3}=0.05[\mathrm{~m}], l_{3}=0.15$ [m]. As seen from the results in Figure 3(b), due to the higher permeability of the Balsa region the flow reaches to the bottom of the domain faster than it moves in the preform region. Consequently, a part of the preform region is not fully saturated by the resin and that is one of the most common issues seen in the manufacturing process [1]. This issue can be solved by increasing the infusion pressure and/or increasing the process time in order to let the preform region get fully wetted by the resin [3]. It should be noted that curing of the resin (which is neglected in this study) also plays a role in the fluidity of the resin and hence the wetting of the preform region.

(a)

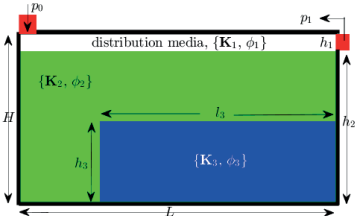

(b)

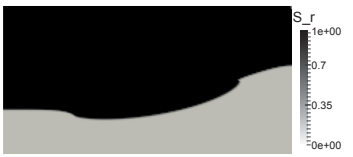

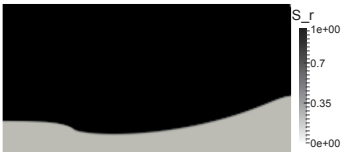

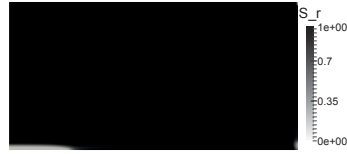

FIGURE 3. Case study for the resin infusion containing a Balsa wood, (a) model concept, and (b) corresponding results.

\section{CONCLUSION}

In this paper, we have used a porous medium method to simulate the resin injection process in manufacturing of composite parts. The model concept is developed and implemented in the open source numerical simulator DuMu ${ }^{\mathrm{x}}$. We have tested the model with both constant and tensorial permeability for the preform (porous) medium and the results showed a reasonable agreement with the ones presented in similar studies [1, 2, 3]. Presence of different regions with different permeability and porosity has also been studied and the results showed that such a layup configuration may lead to an unsaturated region in the domain. Based on the studies conducted in this paper, it is concluded that the developed model can be utilized for qualitative prediction of resin infusion in manufacturing of composite parts, however, proper constitutive laws ( $p_{c}-S_{e}$ and $k_{r \alpha}-S_{e}$ correlation) as well as exact permeability values has to be put in the code.

\section{REFERENCES}

1. N. C. Correia, F. Robitaille, A. C. Long, C. D. Rudd, P. Šimáček, and S. G. Advani, Trans. ASME 126, $210-215$ (2004).

2. K.-T. Hsiao, R. Mathur, S. G. Advani, J. W. G. Jr., and B. K. Fink, J. Manufactur. Sci. Eng. 122, $463-475$ (2000).

3. Q. Govignon, S. Bickerton, J. Morris, and P. A. Kelly, Composites: Part A 39, 1412-1426 (2008).

4. R. Helmig, Multiphase Flow and Transport Processes in the Subsurface: A Contribution to the Modeling of Hydrosystems, Springer, 2011.

5. B. Flemisch, M. Darcis, K. Erbertseder, B. Faigle, A. Lauser, K. Mosthaf, S. Müthing, P. Nuske, A. Tatomir, M. Wolff, and R. Helmig, Adv. Water Res. 25, 1102-1112 (2002).

6. R. Helmig, B. Flemisch, M. Wolff, A. Ebigbo, and H. Class, Adv. Water Res. 51, 52-66 (2013).

7. P. Binning, and M. Celia, Adv. Water Res. 22, 461-478 (1999).

8. R. Brooks, and A. Corey, Hydraulic properties of porous media, Hydrology paper, Colorado State University (1964).

9. P. Bastian, M. Blatt, A. Dedner, C. Engwer, R. Klöfkorn, R. Kornhuber, M. Ohlberger, and O. Sander, Computing 82, 121-138 (2008).

10. M. S. Koefoed, Modeling and Simulation of the VARTM Process for Wind Turbine Blades, Ph.D. thesis, Aalborg University (2003). 